Epoche 1863 Jan. 0, mittl. Zt. Berlin.

$$
\begin{aligned}
& \boldsymbol{H}_{0}=-18^{\circ} 43^{\prime} 34^{\prime \prime} 9 \\
& \pi=58 \quad 9 \quad 1,3 \\
& \left.\Omega=\begin{array}{lll}
2 & 7 & 1,7
\end{array}\right\} \text { M. Aequim. 1863,0. } \\
& i=22755,24 \\
& \varphi=74820,42 \\
& \mu=811^{\prime \prime 568} \\
& \log a=0,4271211 \text {. }
\end{aligned}
$$

\begin{tabular}{|c|c|c|c|}
\hline & \multicolumn{2}{|c|}{$\mathbf{R}-\mathbf{B}$} \\
\hline & & $\Delta \alpha$ & $\Delta \delta$ \\
\hline \multirow[t]{5}{*}{1862} & Nov. 15 & $-2^{\prime \prime} 7$ & $-2^{\prime \prime} 8$ \\
\hline & 26 & $+4,6$ & $-1,9$ \\
\hline & Dec. 16 & $-1,6$ & $+2,6$ \\
\hline & 19 & $-3,4$ & $+4,7$ \\
\hline & 20 & $+1,6$ & $+0,1$ \\
\hline \multirow[t]{3}{*}{1863} & Jan. 18 & $-0,3$ & $+0,5$ \\
\hline & Feb. 15 & $+4,6$ & $-0,4$ \\
\hline & 17 & $-2,9$ & $-3,2$ \\
\hline
\end{tabular}

womit die Ephemeride für das Berliner Jahrbuch 1866 berechnet ist. Die Beobachtungen werden so dargestellt:

Im Nittel ans den verschiedenen Schätzungen wird die Grösse des Planeten in der mittleren Opposition erhalten 13,0. Bemerkenswerth ist indess die Weisse des Lichts, mit der er wie ein Punkt glänzte, verbunden mit einer gewissen Nettigkeit des Bildes. Dieses war sehr auffallend hei der Vergleichung mit der nicht gar fernen Feronia an denselben Abenden, wo also der Zustand der Luft keinen Einfluss auf das Urtheil äussern konnte. Letatere bei ungefahr derselben Grösse erschien mehr verwaschen und in einem blaugrauen Licht.

Hamilton College Observatory, im Mai 1863.

C. H. F. Peters.

\title{
Beobachtungen und Elemente des Cometen III. 1863.
}

\begin{tabular}{|c|c|c|c|}
\hline & Mittl. Zt. Pulkowa & $\cdot x$ of & s. \\
\hline April 26 & $6 \quad 10^{n} 13^{n}+30$ & $0^{\mathrm{h}} 31^{\pi /} 1^{\mathrm{s}} 82$ & $+41^{\circ} 30^{\prime} 32^{\prime \prime} 1$ \\
\hline Mai & 104942,0 & $138 \quad 39,18$ & $+463142,5$ \\
\hline
\end{tabular}

Von Hern Dr. Gyldén auf Pulkowa.

Bei der Bahnlestimmung babe ich folgende Beobachtungen benutzt :

1) die Refractorbeobachtung ron April 16 (A. N. 1410),

2) und 3) zwei Meridiaubeobachtungen, die ich von Dr. Winneck e erhalten habe, nämlich:

4) zwei Beolochtungen, die ich am Kreismikrometer des Heliometers erhielt; diese sind:

$$
\begin{array}{rllll}
\text { Mai } & 15 & 12^{\mathrm{h}} 10^{\mathrm{m}} 31^{\mathrm{s}} & 2^{\mathrm{h}} 54^{\mathrm{m}} 2^{\mathrm{s}} 22+47^{\circ} 26^{\prime} 35^{\prime \prime} 5 \\
-\quad 15 & 123748 & 254 & 25,24 \quad+472630,9
\end{array}
$$

Die mittl. Oerter der Vergleichsterne sind, auf Wolfers'T.R. reducirt: $\quad 2^{\prime \prime} 51^{\prime \prime} 15^{\circ} 92+47^{\prime \prime} 32^{\prime} 42^{\prime \prime} 8$ Oeltz. 3347 .

$$
25118,35+472013,5 \quad-3347 .
$$

Aus diesen heiden beobb. wurde das Mittel genommen.

Hieraus ergab sich nun durch Variation der Abstände folgendes Elementensystem :

$$
\left.\begin{array}{rl}
T=1863 \text { April } 20,89811 \text { mittl. Zt. Berlin } \\
\pi=305^{\prime \prime} 47^{\prime} 6^{\prime \prime} 3 \\
\beta=25010 \quad 35,4 \\
i=852944,8
\end{array}\right\} \text { Mittl. Aeq. } 1863 .
$$

Diese Elemente. stellen die nebenstehenden Beobachtungen wie folut dar:

\begin{tabular}{crcc} 
& & \multicolumn{2}{c}{$\mathbf{R - B}$} \\
& & $d \alpha$ & $d \delta$ \\
April & 16 & $-0^{\prime \prime 5}$ & $0 " 0$ \\
- & 26 & $+3,2$ & $-5,1$ \\
Mai & 4 & $+2,2$ & $-4,0$ \\
- & 15 & $-1,0$ & $+0,5$
\end{tabular}

Schliesslich sind die mittleren Aequatorconstanten:

$$
\begin{aligned}
& x=(9,540427) r \sin \left(337^{\circ} 5355^{\prime \prime} 7+v\right) \\
& y=(9,982419) r \sin (2993520,5+v) \\
& z=(9,990526) r \sin (33 \quad 619,7+v) .
\end{aligned}
$$

Pulk owa, 1863 Juni .

H. Gyldén.

\section{Schreiben des Herrn Goldschmidt an den Herausgeber.}

Mich auf meine Privat-Mittheilung vom $8^{\text {ten }}$ März d. J. beriehend, worin ich die Ehre hatte, thnen von den kleinen Sternen in der Nähe des Sirius zu schreiben, wollie ich abwarten, bis andere Beohachter das von mir Gesehene bestätigt haben würden. Der Mangel eines grossen Ferniohrs liess mich in heständiger Unruhe, und so vergingen die besten Abende, bis Sirius schon zu tief an Horizonte stand, un mit Sicherheit heohachtet werden zu können. Die Gewissheit des Yor- 
handenseins kleiner Sterne hatte mich hewogen, der Akademie der Wissenschatten hieselbst die Anyeige zu machen. Um so viel wie möglich der Täuschung zu cutgehen, die durch falsche Bilder in Fernrohr herorgebracht werden kann, musste ich alwwaten, bis sich für mein Lokal beohachthare, helle Sterne zeigten, um die Prüfung auf dieselbe Beobachtongsart vorzunehmen. So bahe ich werler an Altain noch au Antares dieselbe Erscheiuung der Lichtpunkte wahrgennmmen. Die von Hern Pater Secchi gesehenen Lichtpunkte sind also wahrscheinlich kleine Sterne. Reverend Mr. Dames heobachtete das Sternchen $D$, welches irh zur Zeit der Entdekkung gemessen habe, und dessen ostliche Entternung von Sirius ich ungefälır in $A R=3^{5} 60$ his' $3^{\circ} 80$ fard. Indessen habe ich, der Schwierigkeiten halber, und da ich von Tag zu Tag ein grosses Fernrohr zu meiner Verlïgung erwartete, auf diese Vlessung kein grosses Gewicht gelegt. Auch wird sich noch ein kleineres Steruchen $H$, astlich von $D$, in derselben Parallele finden, welches vielleicht $10^{\prime \prime}$ von $D$ entfernt sein wird, und dessen ich in meituem Briefe an Sie vom 8. März Erwähnung that. Dasselbe war heim Messen des Sternchens $D$ meistens sichtbar.

Durch die von lhuen über Sirius angestellten merkwürdigen Untersuchungen wird diese Sache gewiss um desto interessanter für Sie sein. Ich war nie geneight, alle Sternchen sofort „Begleiter" zu nennen, wic man solches in meinem Namen in verschiedenew Zeitscbriften gethan. Ich hoffe, dass man sich mit Sirius bei seinem Wiedererscheinen ernstlich beschäftigen wird.

Paris, 1863 Mai 23. Hermann Goldschmidt.

\section{Bestimmung der Bahn des Cometen III. 1863, von Hern Dr. Frischauf in Wien.}

$U_{m}$ eine genauere Bahn des Cometen III. 1863 zu erbalten, bildete ich mir mit Hülfe der von Herrn Cöny in den Pariser Bulletins gegebenen Elentente folgende auf das nittlere Aequinoctium 1863,0 bezogene Normalorte:

Zahl Zahl

M. Greenw. Zt. $\underbrace{\text { Mittl. Rert. }} \underbrace{\text { d. Beob. Nittl. Decl. }}$ d. Beob.

April $18,0 \quad 350^{\circ} 21^{\prime} 8^{\prime \prime} 0 \quad 17,+29^{\circ}+2^{\prime} 4^{\prime \prime} 7 \quad 17$

Mai $7,0 \quad 294258,8 \quad 5 \quad+471146,4 \quad 4$

Mai $18,0 \quad 47111,3 \quad 6 \quad+47944,6 \quad 6$

Wi en, 1863 Juni 18 .
Hieraus ergeben sich folgende Elemente:

$\boldsymbol{T}=$ April 20,86067 mittlere Greenw. Zt.

$$
\left.\begin{array}{rl}
\pi & =305^{\circ} 47^{\prime} 24^{\prime \prime}+ \\
\Omega & =2501039,1
\end{array}\right\} \text { mittl. Aeq. } 1863,0
$$

Der mittlere Ort wird dargestellt:

Bcob.-Rechn. $d \lambda=-1^{\prime \prime} 6, \quad d \beta=+4 " 9$.

Dr. Johann Frischauf.

\section{A n z i ge. \\ Verkauf des ehemals Schröter'schen 13 -füssigen Reflectors.}

Wegen Mangels an Raum beabsichtige ich das vorbezeichnete, vor mehren Jahren von der Güttinger Sternwarte von mir angekaufte Instrument für 80 Thaler Preuss. Cour. wieder zu veräussern. Das Instrument, dessen grosser Spiegel 9 Zoll Apertur besitzt, befindet sich, namentlich in seimem optischen Theile, im besten Zustande. Es hat zwei astronomische Oculare und einen 3-füssigen achromatisctien Sucher.

Hannover, 1863 Juni 11.

Kriegsrath Haase.

\section{I l h h a I l.}

(Zu Nr. 1419.) Observations of Comets and small Planets, made at the Observatory of Harv. Coil., Cambridge U. S. A. Conmunicated by Prof. G. P. Bund, Director. 33. - On Leukothea, by E. Schubert. (Communicated by Prof. J. WFinlock, Superintendent of the American Nautical-Almanac.) 41. - Elenente und Ephemeride der Diana (78), von Herrn J. Spengler in Berlin. 47. - Ephemeride des Cometen II. 1863 , von Herrn R. Engrelmann. 47. -

(Zu Nr. 1420.) On the Rate of Ascent of the Envelopes of the Great Comet of 1858. By G. P. Bond, Dir. of the Observatory of Harvard College. 49. - Schreiben des Herrn Prof. Wolf, Directors der Sternwarte in Zürich, an den Herausgeber. 58. - Elemente und Ephemeride des Planeten (65), von Herrn II. Frituche. 61. -

Altona 1863. Juni 26. 